\title{
Research On Arithmetic About Seamless Transformation Positioning Coordinates Interior And Outdoor Based On BDS And RFID
}

\author{
Mingjing Guo ${ }^{* \dagger}+$, Shaofeng Bian*, Xin Xiong* \\ * School of Electrical Engineering(Navy University of Engineering), Wuhan 430033, China; \\ ${ }^{\dagger}$ College of Science(East China University of Technology), Nanchang 330013, China
}

Keywords: BDS; RFID; seamless position; transformation of coordinate; Smart travel

\begin{abstract}
At present due to the indoor positioning is still a dead zone for satellite signal the positioning coordinates of indoor and outdoor are identified separately. And it led to a problem that the seamless fusion positioning can't really come true. This paper proposed a positioning solution that whether it is indoor or outdoor, the coordinate of the unknown terminal could be identified in a unified coordinate system by transformation of coordinates. It described the applicability of the positioning method based on BeiDou satellite navigation system (BDS ) and radiofrequency (RFID) technology in indoor and outdoor scenic spot. And it illustrated the algorithm of rotating and translating the indoor coordinates. This research could realize the seamless fusion positioning of a terminal in indoor or outdoor scenic spot making full use of existing equipment resources in the tourism scenic spots, and provide a new idea for positioning, guide and information pushing based on Location-based services (LBS) in smart travel.
\end{abstract}

\section{Introduction}

With the rapid development of the tourism market and the rise of Smarter Cities construction in our country, the "Smarter travel" arises at the historic moment which making the digital travel that originally emphasized the informationization and quantitative convert to intelligent travel and scenic spots which emphasize personalized and intelligent service construction $^{[1]}$. The key component in smarter travel is information sensing devices such as RFID, GNSS and infrared inductors which can do intelligent identification, location, tracking, monitoring and managemen ${ }^{[2]}$. Although the technology of GNSS can achieve accurate positioning, it can't be used in indoor environment except with the help of other wireless network positioning technologies. So, it is an inexorable development trend in tourism industry which providing integration positioning service for tourists using RFID indoor and GNSS outdoor to achieve seamless fusion positioning. And with the construction and improvement of Beidou navigation system which is independent research and development, interior and outdoor seamless positioning using the BDS is becoming the most important orientation. It is also the hottest research field in positioning technology.
The concept of indoor and outdoor positioning is a service to rapidly obtain location information both in environment indoor and outdoor for providing real-time location and tracking. At present, positioning technology research mainly concentrated in the following respects including single outdoor positioning, single indoor positioning, combination outdoor positioning, fuse positioning between indoor and outdoor and so on, and the last one is the ideal solving direction for meet the need of positioning. Based on the Global Positioning System (short for GPS) and wireless sensor network the localization research has always been a hot direction of the study on the orientation of indoor and outdoor $^{[3,4,5]}$. With China's BeiDou satellite navigation system (BeiDou navigation satellite system, BDS), the upgrade of BDS into Location service (Location -based service, LBS) market, indoor and outdoor seamless locating method based on the BDS began to emerge. In terms of the present research status, it is in the majority that research on application of BDS mix with Bluetooth or with Wi-Fi technology ${ }^{[6,7]}$. The indoor positioning using Wi-Fi technology is based on fingerprint which come from a fingerprint database constructed early offline survey and needed continuously update late. The cost is high and the signal is not stable, seriously affecting the effects of indoor location. Comparing to Wi-Fi technology, the Bluetooth is more stable, but need a fingerprint database constructed early, too. So it is not fit for tourist attraction environment. Popularity of RFID technology in the tourism industry, greatly promoted the foundation of the "wisdom tourism" construction, especially in the ticketing, tourism commodity management, scenic spot recognition, ${ }^{[8]}$ etc.. Therefore, this letter try to use the BDS and now relatively mature RFID automatic identification technology to construct a seamless positioning model fusing indoor and outdoor scenic spots from the perspective of availability in order to implement the intelligent management for the scenic area.

\section{Theoretical basis and assumption}

Smart tourism is to provide tourists with integrated location service in scenic. It can realize the seamless positioning switching between indoor and outdoor to display visually completed feature information of the indoor and outdoor scenic spot in user's terminals. LANDMARC ${ }^{[9]}$ is online measurement scheme based on RFID technology, with the method of online measuring to create signal fingerprint databases. This measurement can satisfy for the need of 
online automatic positioning for multiple tracks (tags). In view of the wide application of RFID technology in the tourism industry, it is a better choice to realize the indoor scenic location tracking. However, due to the layout of reference labels affect directly the positioning performance of tracking labels, LANDMARC is not variable in fickle outdoor scenic environment. BDS can accurately position the location of tourists in outdoor scenic. Compared with GPS technology, the unique two-way short message communication function of Beidou can realize location information and remote information interaction not depend on communication network. Especially for some poor scenic outdoor mobile communication network or mobile communication can't cover, under the condition of power outages, etc. ${ }^{[10,11]}$. BDS could have crucial advantages. Therefore, this article should still adhere to the method of integrating satellite positioning technology using outdoor and RFID positioning technology using indoor to give full play to the advantages of both technologies, and try to get a consistent coordinate system expression for location information of indoor and outdoor. The emphasis of this paper is how to transform outdoor space coordinates (or geodetic coordinates) and indoor coordinates (local coordinates)by reasonable way, so as to realize seamless location coordinates switch between indoor and outdoor scenic spot under the circumstance of keeping tourists terminal equipment and the existing positioning facilities not increased as far as possible.

Assumptions are as follows: the scenic area consists of an indoor attraction, and the indoor attractions for single layer structure, namely not consider problems of floor switch in multi-story buildings about indoor positioning. The outdoor positioning information is three-dimensional space geodetic coordinates (or space rectangular coordinate) based on the three dimensional space geodetic coordinate system coming from satellite positioning signals through Beidou terminals. The indoor positioning information through LANDMARC online measurement is two-dimensional Cartesian coordinates based on the engineering coordinate system of indoor local space. So this article would transform engineering coordinates indoor into two-dimensional station coordinates, and then convert the local coordinate system into three-dimensional space geodetic coordinate system by coordinate system translation, so as to realize unified expression of indoor and outdoor dynamic location data.

\section{Model and arithmetic}

Because the setting of indoor local coordinate systems is generally according to the layout of the reference tags. It is not the same direction as outdoor coordinate systems, and has some flexibility. To achieve the unity expression of the indoor and outdoor location information, first of all, you should rotate the indoor local coordinate system to make indoor and outdoor coordinate systems in the same direction, and keep the origins of the two coordinate systems coinciding. And then you still need move the new coordinate system by means of coordinate translation on WGS84 coordinate system origin. Specific algorithms are as follows.

\subsection{Obtain engineering coordinate indoor}

LANDMARC is online measurement program based on RFID technology, with the method of online measuring to create signal fingerprint databases ${ }^{[12]}$. In addition to tracking tags, LANDMARC also set up reference tags as reference points in the positioning systems. This article assumed that there are $n$ readers, $m$ reference tags and $u$ tracking tags. For a tracking tag needed to solve the signal intensity vector can be denoted as $S=\left(S_{1}, S_{2}, \cdots, S_{n}\right)$, and the signal intensity vector of a reference tag can be denoted as $\theta=\left(\theta_{1}, \theta_{2}, \cdots, \theta_{n}\right)$. The Euclidean distance between the tracking tag needed to solve and the No. $i$ reference tag in the signal intensity space is

$$
E_{j}=\sqrt{\sum_{i=1}^{n}\left(\theta_{i}-S_{i}\right)^{2}}, j \in(1, m)
$$

The engineering coordinate of the point $P$ needed to be measured is just the location of the tracking tag which can be obtained by weighted averaging the coordinates of the first $k$ most nearest reference tags. The formula is

$$
(x, y)=\sum_{i=1}^{k}\left\lfloor\left(\frac{1 / E_{i}^{2}}{\sum_{i=1}^{k} 1 / E_{i}^{2}}\right) \bullet\left(x_{i}, y_{i}\right)\right\rfloor
$$

\subsection{Rotate direction of coordinate system}

To make the anchor point achieve display on the map, map projection is required. Most inland scenic spots in our country generally choose gauss projection to establish basic principal scale topographic maps. It means that in the gauss projection plane, the projection of the intersection of the central meridian and the equator is as natural origin point while the projection of central meridian as $\mathrm{Y}$-axis and the equator as $\mathrm{X}$ axis commonly used to say $X$ and $Y$ which constitute the Gauss-Krueger plane rectangular coordinate system ${ }^{[13,14]}$. In view of the small regional scope of most tourist attractions, deformation problems of gauss projection with the difference of longitude don't have to worry about for the time being. Assume the indoor engineering rectangular coordinate system is $A O^{\prime} B$ according to the layout of indoor reference tags. To make the original point of coordinate system $X^{\prime} O^{\prime} Y^{\prime}$ and $A O^{\prime} B$ coincide, it need Shift the original point of outdoor coordinate system to the indoor coordinate system. Assume the angular separation $\angle B O^{\prime} Y$ is $\beta$ which can be measured, the point $P\left(x_{1}, y_{1}\right)$ in the indoor engineering rectangular coordinate system is $P\left(x_{2}, y_{2}\right)$ in the new coordinate system. The rotating is shown in Fig. 1. 


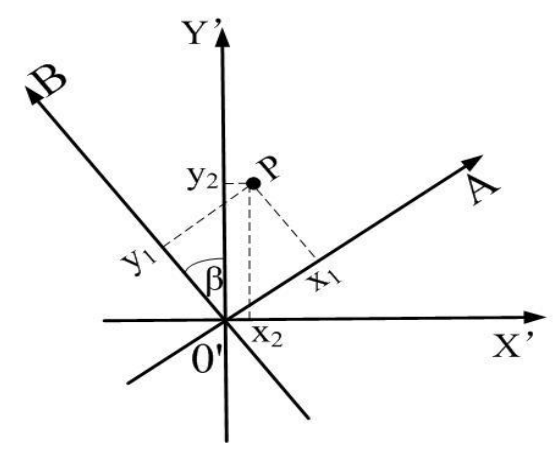

Fig.1 The rotating of indoor coordinate system

The relationship between the point of $P\left(x_{1}, y_{1}\right)$ and

$$
\begin{aligned}
& P\left(x_{2}, y_{2}\right) \text { is } \\
& \left\{\begin{array}{l}
x_{2}=\sqrt{x_{1}^{2}+y_{1}^{2}} \cos \left(\beta+\operatorname{Arctan} \frac{y_{1}}{x_{1}}\right) \\
y_{2}=\sqrt{x_{1}^{2}+y_{1}^{2}} \sin \left(\beta+\operatorname{Arctan} \frac{y_{1}}{x_{1}}\right)
\end{array}\right.
\end{aligned}
$$

\subsection{Transform engineering coordinates to topocentric coordinates}

For a topocentric coordinate system, it is namely the threedimensional cartesian system which is made up of three axis $U 、 V$ and $W$ orthogonal to each other. The origin is on the earth's surface or near the earth's surface. Axis $U$ points to the east, axis $V$ points to the north and the pointing of axis $W$ conform to the right-hand rule. The most current map of scenic spots is based on two-dimensional map, so the elevation axis won't be considered here ${ }^{[15]}$. And for the limited area of scenic spots, the two-dimensional direction of Gauss-Krueger plane rectangular coordinate system and topocentric coordinate system are basically identical, which is shown in Fig.2. If the point $O$ is the origin of topocentric coordinate system, its coordinate is $U=V=W=0$, and the coordinate of point $O^{\prime}$ is $(u, v)$ in the topocentric coordinate system. The new coordinate in the topocentric coordinate system of point $P\left(x_{2}, y_{2}\right)$ is:

$$
\left\{\begin{array}{l}
x=x_{2}+u \\
y=y_{2}+v
\end{array}\right.
$$

For convenience of calculation, a known coordinate point indoor scenic area can be selected as the point $O^{\prime}$, which can be known survey point before building. A near outdoor point around the scenic spots can also be selected, and its coordinates is transformed from geodetic coordinates measured by satellite positioning.

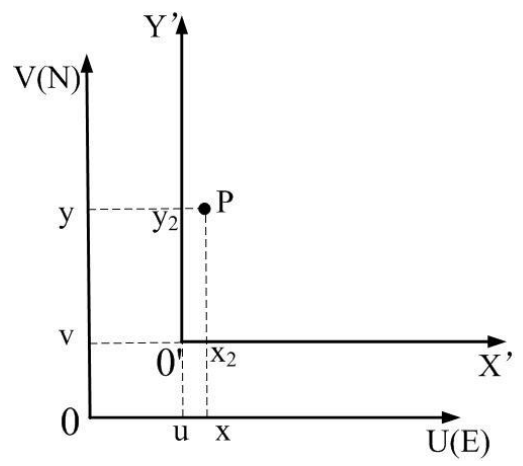

Fig.2 The transforming of coordinate system

\subsection{Transform topocentric coordinates to geodetic coordinates}

The last step is to solve the geodetic coordinates $P(b, l, h)$ of specific indoor point $P(x, y)$. Assume the spatial rectangular coordinate of original point $O$ in topocentric coordinate systems is $\left(X_{0}, Y_{0}, Z_{0}\right)$, and the ellipsoidal height is a constant value $h_{s}$, so the geodetic coordinate of original point $O$ is $\left(B_{0}, L_{0}, h_{\mathrm{s}}\right)$ which is known. And then the spatial rectangular coordinate $\left(X^{*}, Y^{*}, Z^{*}\right)$ of point $P(x, y)$ is as follows:

$$
\left\{\begin{array}{c}
X^{*}=X_{0}-x \sin L_{0}-y \sin B_{0} \cos L_{0}+h_{s} \cos B_{0} \cos L_{0} \\
Y^{*}=Y_{0}+x \cos L_{0}-y \sin B_{0} \sin L_{0}+h_{s} \cos B_{0} \sin L_{0} \\
Z^{*}=Z_{0}+y \cos B_{0}+h_{s} \sin B_{0}
\end{array}\right.
$$

As a result, the indoor positioning information can be transformed into consistent positioning information representation with outdoor. By appropriate map matching algorithm, the specific point indoor and outdoor location can be matched to shows in the same map, so as to realize the attractions indoor and outdoor seamless fusion positioning ${ }^{[16]}$. Also, since the early stage of the construction of the scenic spot the topographic survey data include indoor and outdoor attractions would be obtained. If using grid location attribute auxiliary, the actual positioning area of indoor and outdoor attractions can be get. Moreover combining with the two-way communication function of the Beidou satellite navigation system can also realize personalized information directional push function in attractions, which coincides with the ultimate goal of smart tourism.

\section{Conclusion}

In this paper, based on the development status of smart tourism in our country, the Beidou satellite navigation and positioning technology is introduced into tourism scenic location services system architecture. From the perspective of LBS discusses the positioning methods and techniques applicable to the scenic spot of indoor and outdoor, build the indoor and outdoor seamless fusion positioning model integrated with BDS and RFID technology, especially for the outdoor and indoor positioning coordinates, due to different 
reference frame, the position information of space and time can not unified, through coordinate transformation and the coordinate system translation method is discussed to realize the indoor positioning coordinates are in accordance with outdoor positioning coordinates. In this paper, the research will help to push the BDS in application of tourism industry, and provides a new train of thought for the smart tourism especially LBS positioning, guidance and information push.

\section{Acknowledgements}

This work was supported by Project Supported by Natural Science Foundation of China, No. 41274013.

\section{References}

[1] ZHANG Ling-yun, LI Nao, and LIU Min. "On the Basic Concept of Smarter Tourism and Its Theoretical System” , Tourism Tribune, 27, pp.66-73, (2012).

[2] XU Ye, GUO Tiecheng, “The Influence of IBM 'Smarter Planet' on China” , Forum on Science and Technology in China, no.3, pp. 148-153, (2014).

[3] LIU Shi-run, CUI Zhong-wei, and WEI Ping-ping. "Development of Smart Tourism Mobile APP based on Android System", Electronic Technology\&Software Engineering, no.8, pp. 85-86, (2015).

[4] WANG Yue-jun, WEN Zhao-yu, and WANG Yan-rong. "The intelligent guide services system based on GPS", Guide of Scitech Magazine, no.8, pp. 78, (2011).

[5] REN Ying-li. "Design of Self-guided Tour System for Scenic Spots based on Pocket PC", Informatization Research, 35, no.6, pp. 47-48, (2009).

[6] WEN Hao. "Design of intelligent guide service platform based on the beidou navigation system", Electronic Design Engineering, 21, pp. 103-105, (2013).

[7] CUI Yu, ZHANG Jing-ning. "Application of LBS Based on BDS in Scenic Spot Intelligence Guide System”, Geomatics \& Spatial Information Technology, 36, pp. 98-100, (2013).

[8] WANG Li-bin, HE Xin-long. "Research about Indoor Plsitoning Algorithm based on RFID Technology", Informaiton \& Communications, no.10, pp. 30, (2014).

[9] L.M.Ni, Y.Liu, Y.C.Lau, et al. "LANDMARC: Indoor Location Sensing Using Active RFID”, Wireless Networks, 10 , pp. 701-710, (2004).

[10] YAO Yi-fei, WANG Hao, and ZHAO Dong-fa. " Review of Bei Dou Navigation Satellite System”, Guide of Sci-tech Magazine, no.8, pp. 10, (2011).

[11] LI Jun-feng, "The Analysis between the Bei Dou Satellite Navigation System and the GPS” , Beijing Surveying and Mapping, no.1, pp. 51-52, (2007).
[12] LI Jun-feng, “An Improved Nearest Neighbors Algorithm based on LANDMARC” , Journal of Wuhan University(Natural Science Edition), 5, pp. 255-259, (2013).

[13] ZHANG Xiao-ping, BIAN Shao-feng, and LI Zhong-mei, "Comparisons Between Gauss and Gnomonic Projections in Polar Regions” , Geomatics and Information Science of Wuhan University, 40, pp. 667-672, (2015).

[14] GAO Wei, WU Wen-kai, and YUAN Chao, "Gauss projection coordinate transformation”, Iron \& Steel Technology, no.1, pp.4-6, (2008).

[15] ZHOU Yuan, LIU Yu-xin, LIN Fu-ming, et al, "The Development and Implementation of Intelligent Tourism System Based on Micro Channel Public Platform", Geomatics \& Spatial Information Technology, 38, pp. 77-81, (2015).

[16] WANG Meiling, CHENG Lin, "Study on Map-matching Algorithm for Floating Car", Acta Geodaetica et Cartographica Sinica, 41, pp. 133-138, (2012). 